\title{
Problems on Solving Matrix Aggregation in Group Decision-Making by Glowworm Swarm Optimization
}

\author{
Yaping Li ${ }^{1,2}$ \\ ${ }^{1}$ School of Management, Hefei University of Technology, Hefei 230009, China \\ ${ }^{2}$ Anhui Economic Management Institute, Hefei 230059, China \\ Correspondence should be addressed to Yaping Li; yplifly@126.com
}

Received 19 May 2016; Revised 16 July 2016; Accepted 17 July 2016

Academic Editor: Muhammad N. Akram

Copyright (c) 2016 Yaping Li. This is an open access article distributed under the Creative Commons Attribution License, which permits unrestricted use, distribution, and reproduction in any medium, provided the original work is properly cited.

\begin{abstract}
Judgment matrix aggregation, as an important part of group decision-making, has been widely and deeply studied due to the universality and importance of group decision-making in the management field. For the variety of judgment matrix in group decision-making, the matrix aggregation result can be obtained by using the mode of glowworm swarm optimization. First, this paper introduces the basic principle of the glowworm swarm optimization (GSO) algorithm and gives the improved GSO algorithm to solve the matrix aggregation problems. In this approach, the consistency ratio is introduced to the objective function of the glowworm swarm optimization, thus reducing the subjectivity and information loss in the aggregation process. Then, the improved GSO algorithm is applied to the solution of the deterministic matrix and the fuzzy matrix. The method optimization can provide an effective and relatively uniform aggregation method for matrix aggregation. Finally, through comparative analysis, it is shown that the method of this paper has certain advantages in terms of adaptability, accuracy, and stability to solving the matrix aggregation problems.
\end{abstract}

\section{Introduction}

In social and economic life, group decision-making is widely applied in various management fields, providing support for solving complicated decisions. Therefore, aggregation of expert opinions in group decision-making is of long standing $[1,2]$. At the same time, Zeng et al. gave an applicable example of group decision-making under Web 2.0 environment; it was shown that group decision problem has important significance in both the present and the future [3]. As the application of information technology becomes widely used, expert opinion forms for group decision-making are changing. Forms of the expert judgment matrix mainly include deterministic type and interval type; fuzzy judgment matrix containing deterministic value and interval value is derived. For judgment matrixes of different types, the matrix aggregation method mainly includes additive aggregation, multiplicative aggregation $[4,5]$, operators-based aggregation [6], graph theory-based aggregation [7], and evidence theory-based aggregation [8]. Lu and Guo gave a matrix aggregation scheme based on an undirected connected graph theory. In the scheme, an aggregation matrix with complete consistency is rebuilt by screening more consistent expert opinions according to opinion deviations, thereby obtaining the importance sequence [7]. In 2014, a matrix aggregation algorithm based on spanning tree aggregation operators was proposed by Huang et al. [8], which applies spanning tree aggregation operators to obtain a completely consistent aggregation matrix according to the relations between the judgment matrix and simple undirected graph spanning tree. Zhai and Zhang [9] proposed a matrix aggregation method based on the evidence theory in group judgment. The above methods mainly focus on the problem of aggregation in the deterministic matrix. In addition, the aggregation method for interval judgment matrix is also an important research direction [10, 11]. In 2015, L. Li and J. Li [12] gave an aggregation method for interval judgment matrix, the three-point interval number judgment matrix aggregation is transformed into optimal two-point judgment matrix, and optimal aggregation intervals are synthesized through plant growth simulation algorithm. For Pythagorean fuzzy multiple-criteria decisionmaking problems, Zeng et al. [13] developed a new method 
with aggregation operators and distance measures in 2016. Meanwhile, Zeng et al. [14] presented another aggregation operator that uses generalized means in a unified model between the probability and the OWA operator. Moreover, more attention has also been paid to differential weighting research for expert opinions [15].

Traditional expert judgment matrix aggregation methods are mainly used for direct arithmetic and logic operations based on consistency regulation for the existing matrix. It has higher dependence on the matrix type and quality; thus, reduction of consistency regulation, subjectivity, and information loss in the aggregation process are still key problems for group decision-making in matrix aggregation. The glowworm swarm optimization (GSO) [16] proposed by Krishnanand and Ghose is a new type of swarm intelligence optimization algorithm; it has a higher efficiency than the traditional swarm intelligence algorithms in solving multimodal problems [17]. GSO algorithm is hereby introduced into the matrix aggregation; the expert matrix, as the known feasible solution and the parameter of the objective function, is optimized continuously by GSO algorithm for solving the feasible solution with closer Euclidean distance to the expert matrix as the aggregation result. Therefore it can play a positive role in improving the consistency of the judgment matrix after aggregation, reducing the subjectivity of the aggregation process and the information loss of the original judgment matrix. The researches herein mainly include three parts: Section 2 gives the optimization idea of matrix aggregation-oriented GSO algorithm, Section 3 shows a matrix aggregation method based on an improved GSO algorithm, and Section 4 presents an experimental analysis for deterministic judgment matrix aggregation and interval judgment matrix aggregation.

\section{Matrix Aggregation-Oriented GSO Algorithm}

2.1. Basic Idea of GSO Algorithm. GSO algorithm is to solve the problem mainly through each glowworm representing a feasible solution to the objective problem in space. Glowworms will gather toward high-brightness glowworms by mutual attraction and movement, thus finding multiple extreme points in the solution space for the objective problems. The main idea is described as below.

Step 1 (initialize the algorithm). This includes assigning relevant parameters such as the quantity of glowworms, initial position, initial fluorescein, decision-making radius, fluorescein volatilization rate, and domain change rate.

Step 2 (calculate the fitness according to the objective function). That is, calculate the fitness of each glowworm in place according to the objective function for the specific issues.

Step 3 (determine the moving direction and step length of the glowworm). Each glowworm searches for the glowworm with higher fluorescein value within its own decision-making radius and determines the moving direction and step length of the next step according to the fluorescein value and the distance.
Step 4 (update the position of the glowworm). To be specific, update the position of each glowworm according to the determined moving direction and step length.

Step 5 (update the decision-making radius and the fluorescein of the glowworm). Judge whether the algorithm is terminated, and decide whether to enter into the next iteration.

2.2. Features of Judgment Matrix Aggregation. Expert judgment matrix aggregation is a key element for the effectiveness of group decision-making. The judgment matrix aggregation aims at reasonably and effectively integrating expert opinions, removing opinion deviations to the greatest extent, and obtaining a synthetic judgment matrix with the highest consistency. Therefore, each expert matrix herein can be considered as a suboptimal feasible solution, and the initial distribution of glowworms is optimized. Random distribution of glowworms in the solution space is replaced with probability distribution by taking the initial suboptimal feasible solution as the boundary point, so as to improve the optimizing efficiency for solving the judgment matrix aggregation. The specific optimizing ideas are as follows.

First, narrow the initial distribution field of glowworms; each initial glowworm represents a feasible synthetic judgment matrix. The initial value of the glowworm is assumed to be within the field defined by the known expert matrix.

Assume $I$ as the value range of the element in expert judgment matrix, matrix $A$ as the expert judgment matrix, and matrix $B$ as the synthetic judgment matrix corresponding to the glowworm. Here, the judgment matrix is described according to 1-9 scale method [18]:

$$
\begin{aligned}
I & =\left\{\frac{1}{9}, \frac{1}{8}, \frac{1}{7}, \frac{1}{6}, \frac{1}{5}, \frac{1}{4}, \frac{1}{3}, \frac{1}{2}, 1,2,3,4,5,6,7,8,9\right\}, \\
A^{k} & =\left[a_{i j}^{k}\right]_{n \times n}, \quad a_{i j}^{k} \in I, \\
B & =\left[b_{i j}\right]_{n \times n}, \\
& b_{i j}=\left\{x \mid x \in I, x \in\left[\min \left(a_{i j}\right), \max \left(a_{i j}\right)\right]\right\} .
\end{aligned}
$$

Second, in view of the elements in the synthetic judgment matrix of matrix aggregation not completely restricted by $1-9$ scales (i.e., the value range is not $I$ ), the restrictions to $b_{i j} \in I$ are canceled during initialization of glowworm distribution:

$$
B=\left[b_{i j}\right]_{n \times n}, \quad b_{i j}=\left\{x \mid x \in\left[\min \left(a_{i j}\right), \max \left(a_{i j}\right)\right]\right\} .
$$

Third, add probability distribution factors. The aggregation of expert matrix is probably closer to its mathematical expectation. Therefore, the probability for obtaining the optimal solution is greater if the Euclidean distance to the space point represented by the mathematical expectation is closer. Based on random glowworm distribution, probability distribution is introduced to improve the algorithm convergence speed and optimizing effect. 


\section{Matrix Aggregation Method Based on Improved GSO Algorithm}

Through the above analysis and in combination with the features of group decision-making matrix aggregation, the algorithm (M-GSO) solving the judgment matrix aggregation by using GSO algorithm can be described as follows.

Step 1 (initialize). Assume the judgment matrix is designed according to 1-9 scale method, and provide transformation processing for initial judgment matrixes $A_{1}, A_{2}, A_{3}$, $\ldots, A_{n}$ to facilitate comparison for Euclidean distances. Later, refer to the construction method for the distance matrix proposed by $\mathrm{Lu}$ and Guo [7], and transform $\{1 / 9,1 / 8$, $1 / 7, \ldots, 1 / 3,1 / 2,1,2,3, \ldots, 7,8,9\} 17$ scale values into $1-9$ scales, such as $\{1,2,3,4,5, \ldots, 17\}$ correspondingly; that is,

$$
a_{i j}^{\prime}= \begin{cases}10-\frac{1}{a_{i j}} & a_{i j} \leq 1, \\ 8+a_{i j} & a_{i j}>1 .\end{cases}
$$

Thereby transform $A_{1}, A_{2}, A_{3}, \ldots, A_{n}$ into $A^{(1)}, A^{(2)}, A^{(3)}$, $\ldots, A^{(n)}$.

On this basis, the value range of the synthetic judgment matrix element corresponding to each glowworm can be obtained as follows:

$$
\begin{aligned}
B & =\left\{B_{1}, B_{2}, \ldots, B_{n}\right\}, \\
B_{k} & =\left[b_{i j}^{k}\right]_{n \times n}, \quad b_{i j}^{k} \in\left[\min _{s}\left(a_{i j}^{(s)}\right), \max _{s}\left(a_{i j}^{(s)}\right)\right], \\
\exists B_{k} & =\frac{1}{m} \sum_{i=1}^{m} A_{i}^{(s)} .
\end{aligned}
$$

In the meantime, set the quantity of glowworms $N$, step length $s$, initial fluorescein value $l_{0}$, fluoresce in volatilization rate $\rho$, domain change rate $\beta$, decision domain initial value $\gamma_{0}$, domain threshold $\gamma_{\max }$, and other related parameters in GSO algorithm, as well as the maximum number of iterations and the algorithm termination condition.

Step 2 (set the objective function). $y=\max (f(x))=$ $\max \left(1 / \sum_{i=1}^{n} \sqrt{\left(x-A_{i}^{\prime}\right)^{2} \times \mathrm{CI}}\right)$, in which the objective function is introduced into CI to express the consistency ratio of the ith expert judgment matrix:

$$
\mathrm{CI}=\frac{\lambda_{\max }-n}{n-1}
$$

where $\lambda_{\max }$ is the maximum eigenvalue of the judgment matrix:

$$
\lambda_{\max }=\sum_{i=1}^{n} \frac{A W_{i}}{n W_{i}} .
$$

Different judgment matrixes have different consistencies, and the importance is different. The greater the CI, the worse the consistency, leading to the smaller weight of the corresponding judgment matrix.
Meanwhile, introduce corresponding average random consistency index RI and the consistency ratio CR [19]:

$$
\mathrm{CR}=\frac{\mathrm{CI}}{\mathrm{RI}}
$$

If $\mathrm{CR}<0.1$, it is considered that the judgment matrix has consistency, and the element weight result is acceptable. Otherwise, the judgment matrix must be subjected to necessary correction until CR $<0.1$.

Step 3 (calculate the fitness). To be specific, put the position value of each glowworm $B_{1}, B_{2}, B_{3}, \ldots, B_{k}$ into the objective function to calculate the fitness of each glowworm:

$$
f\left(B_{i}\right)=\frac{1}{\sum_{i=1}^{n} \sqrt{\left(B_{i}-A_{i}^{\prime}\right)^{2} \times \mathrm{CI}}} .
$$

Step 4 (update the position of the glowworm). Search for the glowworm $B_{i}$ with the highest fluorescein within the field range $\gamma$ of the glowworm $B_{i}^{\prime}$, in order to determine the direction of $B_{i}$ moving toward $B_{i}^{\prime}$ :

$$
B_{i}=B_{i}+\frac{\left(B_{i}^{\prime}-B_{i}\right)}{\left|\left(B_{i}^{\prime}-B_{i}\right)\right|} \times s .
$$

Step 5 (update the fluorescein). $l_{i}(t)$ represents the fluorescein value of the $i$ th glowworm in the $t$ th iteration, $\rho$ represents the fluorescein volatilization rate, and $\lambda$ represents the extract ratio of the fitness:

$$
l_{i}(t+1)=(1-\rho) l_{i}(t)+\lambda f\left(x_{i}(t)\right) .
$$

Step 6 (update the decision domain). $\gamma_{i}(t)$ represents the decision-making radius of the $i$ th glowworm in the $t$ th iteration, $\beta$ represents the domain change rate, $n_{t}$ represents the quantity threshold of glowworms in the domain, and $N_{i}(t)$ represents the quantity of glowworms within the decisionmaking radius of the $i$ th glowworm in the $t$ th iteration:

$$
\gamma_{i}(t+1)=\gamma_{i}(t)+\beta\left(n_{t}-N_{i}(t)\right) .
$$

Step 7. Judge the number of iterations and termination conditions and either terminate the algorithm or turn to Step 3.

\section{Experimental Analysis}

Deterministic judgment matrix aggregation and interval judgment matrix aggregation are discussed herein. The deterministic judgment matrix is mainly represented as the matrix elements, which are deterministic values, while the element of the interval judgment matrix is a numerical interval. Herein, M-GSO algorithm is compiled by using MATLAB. Referring to the parameter design for GSO algorithm in relevant reference documents and combining actual conditions of the matrix aggregation, relevant parameters for M-GSO algorithm are selected as follows: $N=50, \rho=0.4, \lambda=$ $0.6, \beta=0.08, s=0.1, l_{0}=5, n_{t}=6$, with the maximum number of iterations as 100 . 
4.1. Aggregation for the Deterministic Judgment Matrix. The aggregation method of graph theory, proposed by Lu and Guo, focuses on aggregation for the deterministic judgment matrix. The basic idea of the matrix aggregation scheme based on undirected connected graph theory is to first select $N$ 1 positions in the upper triangle (or lower triangle) of the matrix $N \times N$ based on the basic theory of the undirected connected graph during aggregation for $m N \times N$ judgment matrixes $M_{1}, M_{2}, \ldots, M_{m} \cdot\left\{M_{1}(i, j), M_{2}(i, j), \ldots, M_{m}(i, j)\right\}$ obtained correspondingly in each position $(i, j)$ of $N$ 1 positions is required to be kept relatively consistent, with deviation as small as possible (where the method for deviation calculation is the sum of the absolute values from subtraction between two elements in vectors). Then, calculate the arithmetic mean (additive) or geometric mean (multiplicative) for the elements $A_{i j}$ in these positions. At last, on this basis, according to the principle of proportional rows, calculate the element values in other positions, thereby building judgment matrix with complete consistency.

Four expert judgment matrixes are selected as the deterministic judgment matrix for M-GSO algorithm experiment (shown below):

$$
\begin{aligned}
A_{1} & =\left[\begin{array}{llll}
1 & 7 & 5 & 4 \\
\frac{1}{7} & 1 & 1 & \frac{1}{2} \\
\frac{1}{5} & 1 & 1 & \frac{1}{3} \\
\frac{1}{4} & 2 & 3 & 1
\end{array}\right], \\
A_{2} & =\left[\begin{array}{llll}
1 & 6 & 7 & 5 \\
\frac{1}{6} & 1 & 1 & 1 \\
\frac{1}{7} & 1 & 1 & 1 \\
\frac{1}{5} & 1 & 1 & 1
\end{array}\right], \\
A_{3}= & {\left[\begin{array}{llll}
1 & 6 & 8 & 4 \\
\frac{1}{6} & 1 & 1 & \frac{1}{2} \\
\frac{1}{8} & 1 & 1 & 1 \\
\frac{1}{4} & 2 & 1 & 1
\end{array}\right], }
\end{aligned}
$$

For the above expert matrixes, some judgment matrixes with complete consistency constructed by the aggregation method of graph theory can be obtained, one of which is as below:

$$
A^{*}=\left[\begin{array}{cccc}
1 & \frac{11}{2} & \frac{77}{16} & \frac{55}{24} \\
\frac{2}{11} & 1 & \frac{7}{8} & \frac{5}{12} \\
\frac{16}{77} & \frac{8}{7} & 1 & \frac{10}{21} \\
\frac{24}{55} & \frac{12}{5} & \frac{21}{10} & 1
\end{array}\right] .
$$

The weight result obtained through the aggregation method of graph theory is $W=(0.5476,0.0996,0.1138$, $0.2390)$, and the importance sequence of corresponding elements is $u_{1}>u_{4}>u_{3}>u_{2}$, in which the consistency ratio of $A^{*}$ is $\mathrm{CR}=0$.

According to the above analysis, the original judgment matrix is first transformed into the distance matrix by $\mathrm{M}$ GSO algorithm, as below:

$$
\begin{aligned}
& A^{(1)}=\left[\begin{array}{cccc}
9 & 15 & 13 & 12 \\
3 & 9 & 9 & 8 \\
5 & 9 & 9 & 7 \\
6 & 10 & 11 & 9
\end{array}\right] \text {, } \\
& A^{(2)}=\left[\begin{array}{cccc}
9 & 14 & 15 & 13 \\
4 & 9 & 9 & 9 \\
3 & 9 & 9 & 9 \\
5 & 9 & 9 & 9
\end{array}\right] \text {, } \\
& A^{(3)}=\left[\begin{array}{cccc}
9 & 14 & 16 & 12 \\
4 & 9 & 9 & 8 \\
2 & 9 & 9 & 9 \\
6 & 10 & 9 & 9
\end{array}\right] \text {, } \\
& A^{(4)}=\left[\begin{array}{cccc}
9 & 11 & 10 & 9 \\
7 & 9 & 8 & 7 \\
8 & 10 & 9 & 10 \\
9 & 11 & 8 & 9
\end{array}\right] \text {. }
\end{aligned}
$$

By running the M-GSO algorithm five times with randomized initial points, five synthetic judgment matrixes with complete consistency can be obtained as below: 
TABLE 1: Aggregation for the deterministic judgment matrix.

\begin{tabular}{lcc}
\hline Synthetic judgment matrixes & Optimal weight & Importance sequence \\
\hline$A_{1}^{*}$ & $0.4455,0.1099,0.1875,0.2570$ & $u_{1}>u_{4}>u_{3}>u_{2}$ \\
$A_{2}^{*}$ & $0.4680,0.1085,0.1804,0.2431$ & $u_{1}>u_{4}>u_{3}>u_{2}$ \\
$A_{3}^{*}$ & $0.4620,0.1092,0.1785,0.2502$ & $u_{1}>u_{4}>u_{3}>u_{2}$ \\
$A_{4}^{*}$ & $0.4444,0.1105,0.1891,0.2559$ & $u_{1}>u_{4}>u_{3}>u_{2}$ \\
$A_{5}^{*}$ & $0.4449,0.1105,0.1900,0.2545$ & $u_{1}>u_{4}>u_{3}>u_{2}$ \\
Arithmetic mean & $0.4530,0.1098,0.1851,0.2521$ & $u_{1}>u_{4}>u_{3}>u_{2}$ \\
\hline
\end{tabular}

$$
\begin{aligned}
& A_{1}^{*}=\left[\begin{array}{cccc}
1 & 4.05 & 2.38 & 1.73 \\
0.25 & 1 & 0.59 & 0.43 \\
0.42 & 1.71 & 1 & 0.73 \\
0.58 & 2.34 & 1.37 & 1
\end{array}\right], \\
& A_{2}^{*}=\left[\begin{array}{cccc}
1 & 4.31 & 2.59 & 1.93 \\
0.23 & 1 & 0.60 & 0.45 \\
0.39 & 1.66 & 1 & 0.74 \\
0.52 & 2.24 & 1.35 & 1
\end{array}\right] \text {, } \\
& A_{3}^{*}=\left[\begin{array}{cccc}
1 & 4.23 & 2.59 & 1.85 \\
0.24 & 1 & 0.61 & 0.44 \\
0.39 & 1.63 & 1 & 0.71 \\
0.54 & 2.29 & 1.40 & 1
\end{array}\right], \\
& A_{4}^{*}=\left[\begin{array}{cccc}
1 & 4.02 & 2.35 & 1.74 \\
0.25 & 1 & 0.58 & 0.43 \\
0.43 & 1.71 & 1 & 0.74 \\
0.58 & 2.31 & 1.35 & 1
\end{array}\right] \text {, } \\
& A_{5}^{*}=\left[\begin{array}{cccc}
1 & 4.03 & 2.34 & 1.75 \\
0.25 & 1 & 0.58 & 0.43 \\
0.43 & 1.72 & 1 & 0.75 \\
0.57 & 2.30 & 1.34 & 1
\end{array}\right],
\end{aligned}
$$

where $A_{1}^{*}, A_{2}^{*}, A_{3}^{*}, A_{4}^{*}, A_{5}^{*}$ satisfy $\mathrm{CR}=0$. The sequence of corresponding weights can be calculated according to the matrix $A_{1}^{*}, A_{2}^{*}, A_{3}^{*}, A_{4}^{*}, A_{5}^{*}$, as shown in Table 1.

As shown in Table 1, the arithmetic mean obtained from five running results according to M-GSO algorithm is $w=(0.4530,0.1098,0.1851,0.2521)$, and the importance sequence is $u_{1}>u_{4}>u_{3}>u_{2}$. The sequence result, consistent with those by the method of graph theory, can be stably obtained through either single running or arithmetic mean of the operation result.

However, this method based on undirected connected graph theory does not get the correct results every time. In this paper, we change the elements $A_{i}(1,3)=(5,7,8,2)$ to $A_{i}(1,3)=(3,2,2,1 / 3)$, while adjusting the value of $A_{i}(3,1)$; a new matrix is shown as below:

$$
\begin{aligned}
& B_{1}=\left[\begin{array}{llll}
1 & 7 & 3 & 4 \\
\frac{1}{7} & 1 & 1 & \frac{1}{2} \\
\frac{1}{3} & 1 & 1 & \frac{1}{3} \\
\frac{1}{4} & 2 & 3 & 1
\end{array}\right], \\
& B_{2}=\left[\begin{array}{llll}
1 & 6 & 2 & 5 \\
\frac{1}{6} & 1 & 1 & 1 \\
\frac{1}{2} & 1 & 1 & 1 \\
\frac{1}{5} & 1 & 1 & 1
\end{array}\right], \\
& B_{3}=\left[\begin{array}{llll}
1 & 6 & 2 & 4 \\
\frac{1}{6} & 1 & 1 & \frac{1}{2} \\
\frac{1}{2} & 1 & 1 & 1 \\
\frac{1}{4} & 2 & 1 & 1
\end{array}\right], \\
& B_{4}=\left[\begin{array}{llll}
1 & 3 & \frac{1}{3} & 1 \\
\frac{1}{3} & 1 & \frac{1}{2} & \frac{1}{3} \\
3 & 2 & 1 & 2 \\
1 & 3 & \frac{1}{2} & 1
\end{array}\right] .
\end{aligned}
$$

For these matrixes, we can get some conflicting results through the aggregation method of graph theory. The conflicting importance sequence of corresponding elements is $u_{1}>u_{4}>u_{3}>u_{2}$ and $u_{4}>u_{1}>u_{3}>u_{2}$. The calculation process is described in detail in another article by the author [20]. At the same time, we can obtain the importance sequence $u_{1}>u_{4}>u_{3}>u_{2}$ stably using the M-GSO algorithm.

4.2. Aggregation for the Interval Judgment Matrix. For the indeterminacy and inconsistency in the judgment matrix, 
the relations between the basic consistency and consensus of group decision-making are discussed. A scheme for solving the importance sequence based on integrated arithmetic mean is given by Zhai and Zhang [9]. Three interval judgment matrixes are shown below:

$$
\begin{aligned}
& A^{(1)}=\left[\begin{array}{ccc}
1 & {[2.0,3.0]} & {[4.0,5.0]} \\
& 1 & {[0.15,0.2]} \\
& & 1
\end{array}\right], \\
& A^{(2)}=\left[\begin{array}{ccc}
1 & {[1.5,2.5]} & {[4.0,5.5]} \\
& 1 & {[0.2,0.25]} \\
& & 1
\end{array}\right] \text {, } \\
& A^{(3)}=\left[\begin{array}{ccc}
1 & {[2.6,3.5]} & {[4.5,5.5]} \\
& 1 & {[0.15,0.18]} \\
& & 1
\end{array}\right] \text {. }
\end{aligned}
$$

According to the aggregation method based on integrated arithmetic mean, the combined judgment matrix $A^{*}$ is the arithmetic mean of the original judgment matrix. Meanwhile, $A^{*}$ is a reciprocal matrix:

$$
\begin{aligned}
A^{*} & =\frac{1}{3} \sum_{k=1}^{3} A^{(k)} \\
& =\left[\begin{array}{ccc}
1 & {[2.03,3.0]} & {[4.17,5.13]} \\
{[0.33,0.49]} & 1 & {[0.17,0.21]} \\
{[0.19,0.24]} & {[4.76,5.88]} & 1
\end{array}\right] .
\end{aligned}
$$

The weight result obtained from aggregation in the above interval judgment matrix by the method based on integrated arithmetic mean is $w_{1}=[0.397,0.637], w_{2}=$ $[0.083,0.116], w_{3}=[0.328,0.487]$, and the importance sequence of corresponding elements is $\left(u_{1}>u_{3}>u_{2}\right)$.

Now, the algorithm accuracy $(\theta)$ will be defined by the arithmetic mean of the length of the weight interval:

$$
\begin{aligned}
\theta_{i} & =\max \left(w_{i}\right)-\min \left(w_{i}\right), \\
\theta_{1} & =0.637-0.397=0.24, \\
\theta_{2} & =0.116-0.083=0.033, \\
\theta_{3} & =0.487-0.328=0.159, \\
\theta & =\frac{1}{3} \sum_{i=1}^{3} \theta_{i}=\frac{1}{3}(0.24+0.033+0.159)=0.144 .
\end{aligned}
$$

The difference between the aggregation for interval judgment matrix and the above deterministic judgment matrix is not just in the transformation of matrix elements from the numerical values into intervals; at the same time, the value range is not according to 17 grades corresponding to 1-9 scales anymore but expanded to the continuous interval of 0-9. The consistency index for the deterministic matrix cannot be used. Thus, the upper and lower limits of the modal interval judgment matrix are split into two deterministic judgment matrixes by M-GSO algorithm; that is,

$$
\begin{aligned}
& A_{1}^{(1)}=\left[\begin{array}{ccc}
1 & 2.0 & 4.0 \\
& 1 & 0.15 \\
& & 1
\end{array}\right], \\
& A_{2}^{(1)}=\left[\begin{array}{ccc}
1 & 3.0 & 5.0 \\
& 1 & 0.2 \\
& & 1
\end{array}\right], \\
& A_{1}^{(2)}=\left[\begin{array}{ccc}
1 & 1.5 & 4.0 \\
& 1 & 0.2 \\
& & 1
\end{array}\right], \\
& A_{2}^{(2)}=\left[\begin{array}{ccc}
1 & 2.5 & 5.5 \\
& 1 & 0.25 \\
& & 1
\end{array}\right], \\
& A_{1}^{(3)}=\left[\begin{array}{ccc}
1 & 2.6 & 4.5 \\
& 1 & 0.15 \\
& & 1
\end{array}\right],
\end{aligned}
$$

The judgment matrix is used as the suboptimal feasible solution for optimization by M-GSO algorithm, and the consistency of the judgment matrix is introduced into the objective function as the parameter, so the influence of the consistency of the initial judgment matrix on the aggregation result is relatively small. Moreover, more optimal feasible solutions can also be obtained by running M-GSO algorithm for the deterministic judgment matrix after the transformation of interval judgment matrix. Also, by running M-GSO algorithm for $A_{1}^{(1)}, A_{1}^{(2)}, A_{1}^{(3)}$ and $A_{2}^{(1)}, A_{2}^{(2)}, A_{2}^{(3)}$ in the above deterministic judgment matrix five times, we can also obtain the two sets of synthetic judgment matrixes with complete consistency $A_{1}^{*}, A_{2}^{*}, A_{3}^{*}, A_{4}^{*}, A_{5}^{*}$ and $B_{1}^{*}, B_{2}^{*}, B_{3}^{*}, B_{4}^{*}, B_{5}^{*}$. The results as shown in Table 2 can be obtained.

The two sets of weight results obtained from the operation results of M-GSO algorithm and the arithmetic mean are considered as feasible solutions of weights for the elements $u_{1}, u_{2}, u_{3}$, thereby obtaining the weight range of elements $u_{1}$, $u_{2}, u_{3}$, with the weight intervals as $w_{1}=[0.592,0.677], w_{2}=$ $[0.151,0.169], w_{3}=[0.154,0.256]$, thus obtaining the importance sequence of $u_{1}>u_{3}>u_{2}$. It is consistent with the weight sequence obtained through the method based on integrated arithmetic mean.

According to the definition of algorithm accuracy, the accuracy $\left(\theta^{\prime}\right)$ of the above weight range can be expressed as follows:

$$
\begin{aligned}
& \theta_{i}^{\prime}=\max \left(w_{i}\right)-\min \left(w_{i}\right), \\
& \theta_{1}^{\prime}=0.677-0.592=0.085,
\end{aligned}
$$


TABLE 2: Aggregation for interval judgment matrix.

Synthetic judgment matrixes

$A_{1}^{*}, B_{1}^{*}$

$A_{2}^{*}, B_{2}^{*}$

$A_{3}^{*}, B_{3}^{*}$

$A_{4}^{*}, B_{4}^{*}$

$A_{5}^{*}, B_{5}^{*}$

Arithmetic mean
Optimal weight of the first group of judgment matrixes

Optimal weight of the second group of judgment matrixes

$0.54004,0.14557,0.31439$
$0.60355,0.16131,0.23514$
$0.58768,0.15270,0.25962$
$0.59078,0.15350,0.25572$
$0.63984,0.14281,0.21734$
$0.59238,0.15118,0.25644$

$0.67608,0.16878,0.15514$
$0.67654,0.16903,0.15443$
$0.68154,0.16772,0.15074$
$0.67602,0.16907,0.15491$
$0.67606,0.16903,0.15491$
$0.67725,0.16872,0.15402$
$\theta>\theta^{\prime}$

The following conclusions can be obtained: $\theta_{i}>\theta_{i}^{\prime}$ and

Compared with $w_{1}=[0.397,0.637], w_{2}=[0.083,0.116]$, $w_{1}=[0.328,0.487]$, the weight intervals $w_{1}=[0.592,0.677]$, $w_{2}=[0.151,0.169], w_{3}=[0.154,0.256]$ obtained from MGSO algorithm are remarkably narrowed on interval size, for example, with higher calculation accuracy.

4.3. Comparative Analyses. The above experimental results show that M-GSO algorithm provides a relative uniform solution for the deterministic judgment matrix aggregation and interval judgment matrix aggregation. The traditional aggregation methods often focus on special judgment matrix type; thus in practical application, consistent judging methods cannot be provided for different expert judgment ways of the same problem. A uniform solution can be provided by M-GSO algorithm because the expert judgment matrix is considered as the feasible solution in M-GSO algorithm, and more optimal feasible weight solutions can be obtained through optimization, while traditional matrix aggregation methods are often to operate the judgment matrix; hence, there are more requirements on the expert matrix.

4.3.1. Algorithm Robustness. The expert judgment matrix is subjected to differentiation by M-GSO algorithm. Different expert matrixes have different subjective recognition, and the actual qualities are different; for example, expert judgment matrixes with poor quality have negative impact on the final weight result. Thus, M-GSO algorithm is to differentiate expert matrixes by adding the consistency index of the judgment matrix to the objective function, consequently to weaken the negative impact of the expert matrix with poor consistency on the final result, and meanwhile to further reduce the dependency of matrix aggregation scheme on the consistency of the initial matrix.

4.3.2. Algorithm Stability. Compared with the method of graph theory, the operation results of M-GSO algorithm are more stabilized. According to the specific case analysis for the method of graph theory, the weight sequence results are instable when multiple element values are equal in the distance deviation matrix, but the weight sequence results are obtained through constant optimizing in M-GSO algorithm, so those results are more stable, which is the same as proved above.

4.3.3. Algorithm Accuracy. By defining the algorithm accuracy, the advantages and disadvantages of the weight range can be compared more intuitively. Due to $\theta_{i}>\theta_{i}^{\prime}$ and $\theta>\theta^{\prime}$ in Section 4.2, the accuracy of weight interval of M-GSO algorithm is higher. Therefore, compared with the arithmetic mean, it is more optimal for selecting optimal solutions from multiple poles. For weight results in practical application, the smaller the weight interval, the more accurate the provided decision-making support; for example, the larger the weight interval, the smaller the actual significance. Final weight results are obtained through searching for feasible solutions and using feasible intervals among feasible solutions by $\mathrm{M}$ GSO algorithm, thereby obtaining more effective weight intervals.

\section{Summary}

The result comparison of different decision alternatives or decision factors is usually given in the form of a judgment matrix in group decision-making. The aggregation of the matrix is equivalent to the synthesis of various expert opinions. In this paper, the weight or importance sequence obtained by the M-GSO algorithm can be understood as the importance of decision scheme or decision factors in the specific application. This is the application value of the MGSO algorithm in group decision-making.

This paper presents the M-GSO algorithm to solve the matrix aggregation problems. Unlike the traditional matrix aggregation methods, this method not only can be used in deterministic matrix, but also can be used for the interval matrix. The aggregation mode of M-GSO algorithm is no longer to conduct operation for the judgment matrix itself but to take the judgment matrix as the restrictions for optimization. In the meantime, consideration is given to the quality difference of judgment matrixes in M-GSO algorithm. It is unnecessary to manually regulate the judgment matrixes with relatively poor consistency; thus this optimization is more adaptive. Through comparative analysis, the M-GSO 
algorithm has good robustness, stability, and computational accuracy.

In addition, the effects of random distribution of glowworm position can also be noticed. The M-GSO algorithm should be improved in convergence and accuracy, so that it can be better applied to solve group decision-making problems.

\section{Competing Interests}

The author declares no competing interests.

\section{Acknowledgments}

The work was supported by the fund of the Provincial Excellent Young Talents of Colleges and Universities of Anhui Province (no. 2013SQRW115ZD) and the fund of the Natural Science of Colleges and Universities of Anhui Province (no. KJ2016A162).

\section{References}

[1] C.-L. Hwang and K. P. Yoon, Multiple Attribute Decision Making: Methods and Applications, vol. 186 of Lecture Notes in Economics and Mathematical Systems, 1981.

[2] G. Stasser and W. Titus, "Pooling of unshared information in group decision making. Biased information sampling during discussion," Journal of Personality and Social Psychology, vol. 48, no. 6, pp. 1467-1478, 1985.

[3] S. Zeng, J. Gonzalez, and C. Lobato, "The effect of organizational learning and Web 2.0 on innovation," Management Decision, vol. 53, no. 9, pp. 2060-2072, 2015.

[4] T. L. Saaty, Decision Making for Leasers, Wadsworth Inc, Belmont, Calif, USA, 1982.

[5] E. U. Choo and W. C. Wedley, "Comparing fundamentals of additive and multiplicative aggregation in ratio scale multicriteria decision making," Open Operational Research Journal, vol. 2, pp. 1-7, 2008.

[6] I. Beg and T. Rashid, "A geometric aggregation operator for decision making," Vietnam Journal of Computer Science, vol. 2, no. 4, pp. 243-255, 2015.

[7] Y.-J. Lu and X.-R. Guo, "Effective aggregation method for the AHP judgement matrix in group decision-making," System Engineering Theory and Practice, vol. 27, no. 7, pp. 132-136, 2007.

[8] C. Huang, B. Jiao, F. Huang et al., "An aggregation method for group AHP judgment matrices based on the evidence theory," Science Technology and Engineering, vol. 14, no. 15, pp. 1-4, 2014.

[9] X. Zhai and X. Zhang, "The methods on aggregation of interval number judgment matrixes and calculation of its priorities in the group decision-making," Systems Engineering, vol. 23, no. 9, pp. 103-107, 2005.

[10] E. Han, P. Guo, and J. Zhao, "Method for multi-attribute group decision making based on interval grey uncertain linguistic assessment," Journal of Frontiers of Computer Science \& Technology, vol. 10, no. 1, pp. 93-102, 2016.

[11] E. K. Zavadskas, J. Antucheviciene, S. H. Razavi Hajiagha, and S. S. Hashemi, "The interval-valued intuitionistic fuzzy MULTIMOORA method for group decision making in engineering," Mathematical Problems in Engineering, vol. 2015, Article ID 560690, 13 pages, 2015.
[12] L. Li and J. Li, "Study of three point interval number judgment matrix decomposition and aggregation based on fermat and algorithm," Mathematics in Practice and Theory, no. 17, pp. 214221, 2015

[13] S. Zeng, J. Chen, and X. Li, "A hybrid method for Pythagorean fuzzy multi-criteria decision making," International Journal of Information Technology \& Decision Making, vol. 15, no. 2, pp. 403-422, 2016.

[14] S. Zeng, W. Su, and C. Zhang, "Intuitionistic fuzzy generalized probabilistic ordered weighted averaging operator and its application to group decision making," Technological and Economic Development of Economy, vol. 22, no. 2, pp. 177-193, 2016.

[15] J. M. Merigó, D. Palacios-Marqués, and S. Zeng, "Subjective and objective information in linguistic multi-criteria group decision making," European Journal of Operational Research, vol. 248, no. 2, pp. 522-531, 2016.

[16] K. N. Krishnanand and D. Ghose, "Glowworm swarm optimization for simultaneous capture of multiple local optima of multimodal functions," Swarm Intelligence, vol. 3, no. 2, pp. 87124, 2009.

[17] N. Zainal, A. M. Zain, N. H. M. Radzi et al., "Glowworm swarm optimization (GSO) for optimization of machining parameters," Journal of Intelligent Manufacturing, vol. 27, no. 4, pp. 797-804, 2016.

[18] N. Kotani and Y. Kodono, "A study on the pairwise comparison scale in AHP," Journal of International Studies, vol. 19, pp. 1-13, 2006.

[19] M. T. Escobar, J. Aguarón, and J. M. Moreno-Jiménez, "Some extensions of the precise consistency consensus matrix," Decision Support Systems, vol. 74, pp. 67-77, 2015.

[20] Y. Li, "Optimization of matrix aggregation method based on undirected connection graph theory," Journal of Chongqing Technology and Business (Natural Sciences Edition), vol. 31, no. 5, pp. 10-13, 2014. 


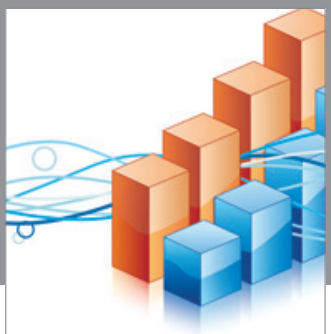

Advances in

Operations Research

vatem alat4

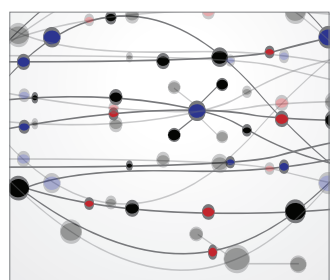

\section{The Scientific} World Journal
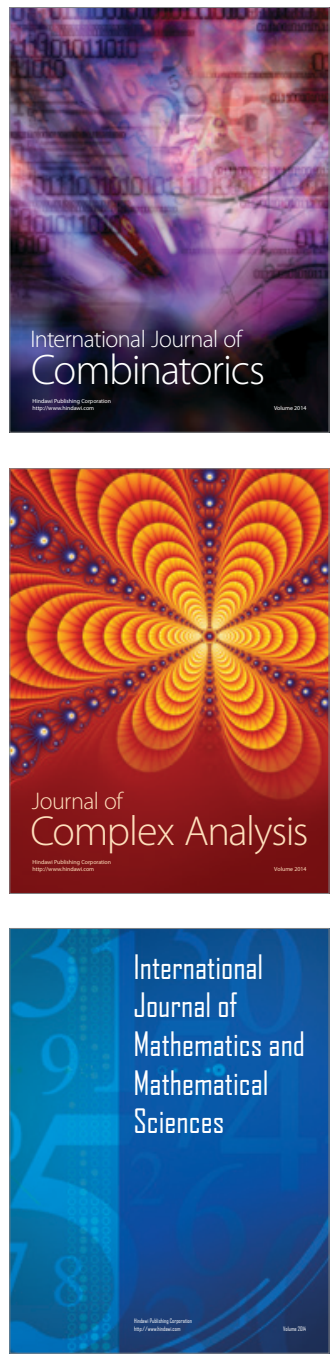
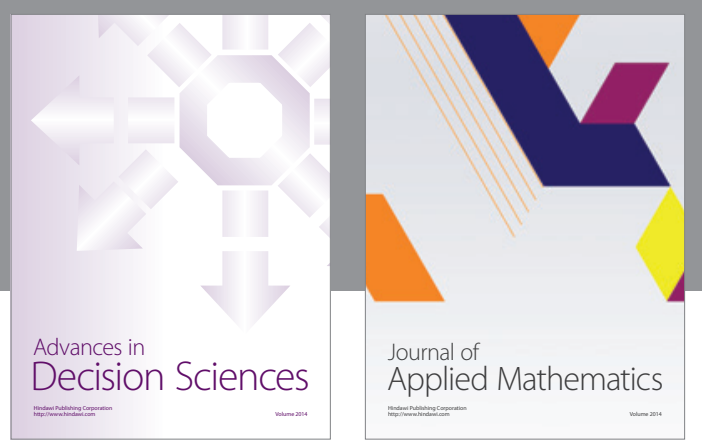

Algebra

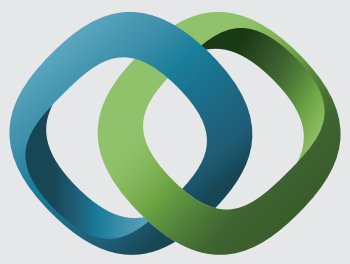

\section{Hindawi}

Submit your manuscripts at

http://www.hindawi.com
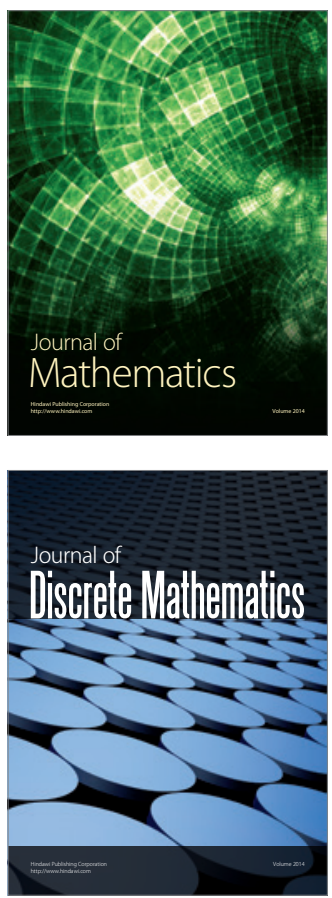

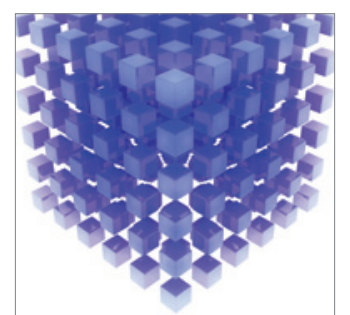

Mathematical Problems in Engineering
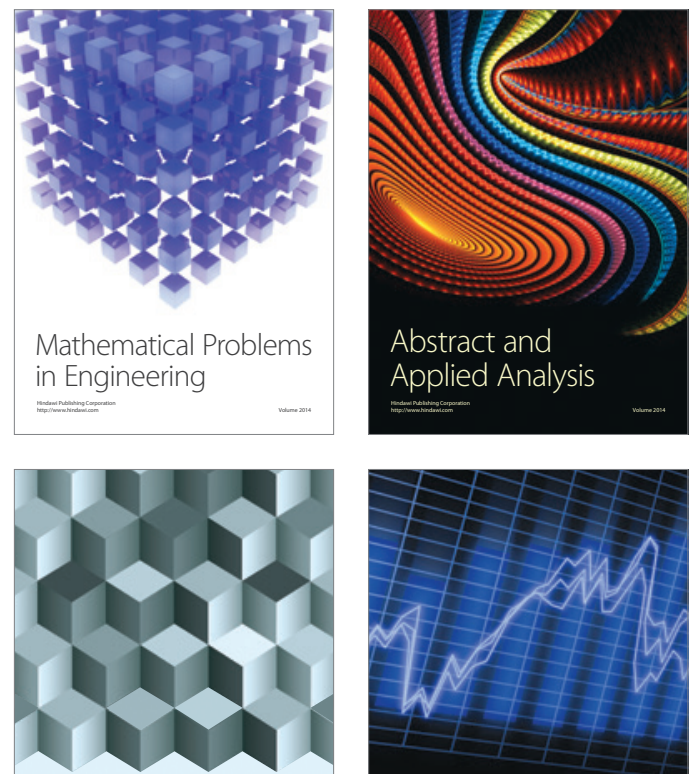

Journal of

Function Spaces

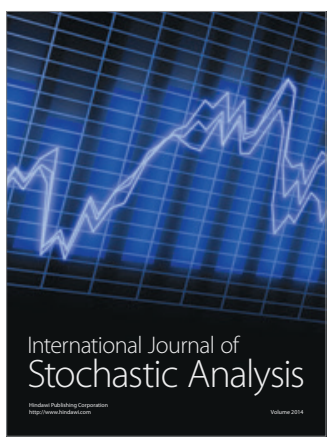

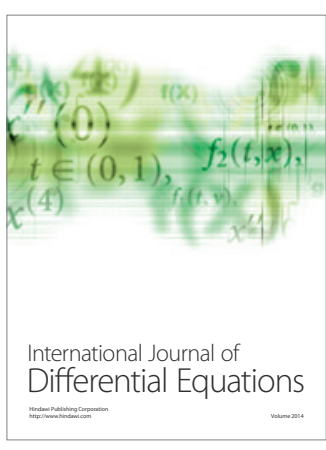
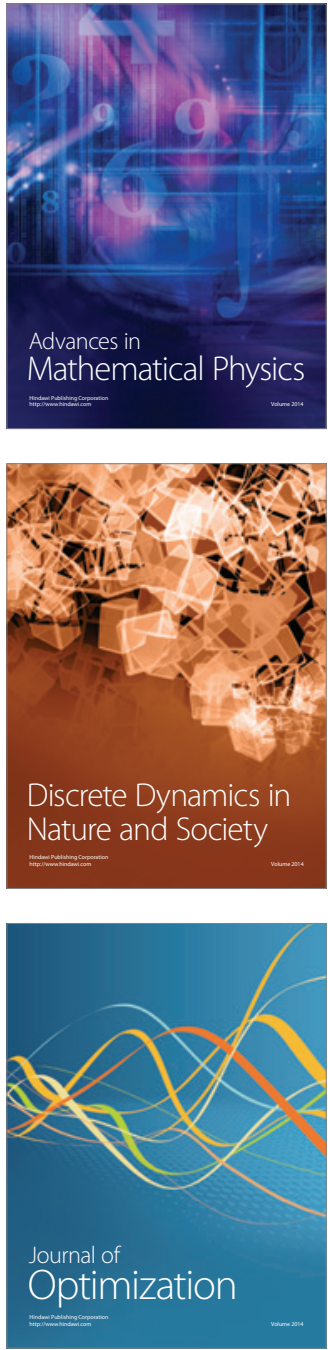